\title{
Investigation and Application of High Megavoltage X-Ray Imaging Mode in Radiotherapy
}

\author{
Quanshi Zhang, Xiwen Wang, Qiyin Sun, Yuehui Jin, Yun Li, Ziyu Li, Tao Sun, Liang Wang \\ The Cancer Affiliated Hospital of Soochow University American Fox Chase Wuxi Cancer Center, Wuxi, China \\ Email: zqs1962@aliyun.com
}

Received 28 October 2015; accepted 14 February 2016; published 17 February 2016

Copyright (C) 2016 by authors and Scientific Research Publishing Inc.

This work is licensed under the Creative Commons Attribution International License (CC BY). http://creativecommons.org/licenses/by/4.0/

(c) () Open Access

\begin{abstract}
After drawbacks and shortages of using conventional $\mathrm{kV}$ or $\mathrm{MV}$ imaging mode were analyzed, this study proposes a new position verification mode with using the energy larger than $15 \mathrm{MeV}$ or nominal accelerating potential greater than 25 MV X-Ray. The new position verification mode is named HMV imaging mode. Along with the comparison of theoretical analyses, phantom experiments and clinical results to the original imaging modes, this report is going to demonstrate the HMV imaging mode is superior to traditional $\mathrm{kV}$ and MV imaging modes. This report first theoretically analyzed three main effects of $\mathrm{X}$-ray interacting with medium by numerous equations and compared their mass attenuation coefficient with different types of tissue. X-ray irradiated on a "Catphan 500" cylinder phantom with different energies to verify these theoretical results. Furthermore, based on phantom experiments' results, we have done numerous clinical trials and comparisons with patient's clinical results. The theoretical and experimental results illustrate that the scanned images from HMV mode have a good quality and have ability to identify different tissue components clearly. HMV imaging mode overcomes drawbacks of position verification from both $\mathrm{kV}$ and MV level imaging mode as well as keeping advantages of $\mathrm{kV}$ and $\mathrm{MV}$ imaging mode. The result indicates that HMV is a good position verification mode in radiotherapy.
\end{abstract}

\section{Keywords}

High Energy X-Ray, X-Ray Imaging Mode, Position Verification, Reaction Cross-Section

\section{Introduction}

In today's world, choosing reasonable ways to detect and treat malignant tumor becomes a hot debated issue 
because tumors may threaten human life. Radiotherapy is one of the three significant therapy strategies for the cancer treatment. Now, the radiotherapy has entered new era of accurate treatment with applying 3D conformal and Intensity-modulated therapy (IMRT) [1] [2]. Chen [3] stated tumor's verification image during radiotherapy period is an essential process and Imaging Guide Radiotherapy Therapy (IGRT) is a new developed system for ensuring tumor to be irradiated at precise position. Currently, $\mathrm{kV}$ level imaging mode $(\mathrm{kV})$ and MV level imaging mode (MV) imaging modes are two main X-ray imaging modes for IGRT on linear accelerators for radiation therapy [4] [5]. Their X-ray energy ranges from $60 \mathrm{kV}$ to $120 \mathrm{kV}$ and 4MV to $15 \mathrm{MV}$ respectively. However, both of these modes have unavoidable shortcoming and inadequate.

For radiotherapy equipments with $\mathrm{kV}$ level imaging mode, it has good image quality and contrast between tissues and anatomical structures. However, the $\mathrm{kV}$ imaging mode needs to equip a set of additional $\mathrm{kV} X$-ray generators and bound up with control sections (Figure 1(a)) [6]. These functions not only increase cost of additional control sections and raise unsafe factors during treatment process, but also heighten failure rates of the whole system and increase the system potential failure rate. The main issues of $\mathrm{kV}$ imaging mode are additional radiation dose delivered to patients during the guidance period and the acquired guidance images from this mode have different isocenter from the treatment beam [7].

For radiotherapy equipments with MV level imaging mode, which a Electronic Portal Imaging Device (EPID) mounted on the opposite of the radiation source [8], do not have shortcomings and the insufficiency as in $\mathrm{kV}$ level imaging mode because MV imaging mode uses the same X-ray source for treatment and imaging (Figure 1(b)). The on-board MV level-imaging mode does not increase the cost and unsafe factors, but is easy to correct additional dosage that is introduced from verification imaging. Additionally, along with relative algorithms, doseguided Radiotherapy (DGRT) is able to use with MV level CBCT. When a metal implanted into human, artifacts that produced from MV CBCT is much smaller than it generated from $\mathrm{kV} \mathrm{CBCT}$. Based on these issues, MV level imaging mode would be an ideal mode for IGRT. However, resolving capability contrast between tissues and anatomical structures is not that good as the images acquired from $\mathrm{kV}$ level imaging mode. This is a fatal flaw of the MV mode and easily leads to larger position errors and uncertainty.

Currently, many manufacturers invest more employees, extend material resources and researchers with applying additional software or equipment modifications to improve the image resolving capability and quality for MV level imaging mode. Such plans of redesigning the size of EPID in MV imaging mode aim to collect more photons, combining $\mathrm{kV}$ imaging with optical imaging devices minimizing dose on patients [7] and using a dual energy radiotherapy imager to acquire the scanning images from both $\mathrm{kV}$ and $\mathrm{MV}$ imaging mode [9]. However, there were not effective methods that can significantly improve the image contrast and quality. This was mainly because Compton Effect within the range of $4 \mathrm{MV}$ to $15 \mathrm{MV}$ X-ray interacting with medium is independent of the atomic number of medium and the energy of photon. Therefore, many manufacturers (i.e. Elekta and Varian [9] and Siemens [10] are forced to use both MV level imaging mode and $\mathrm{kV}$ level imaging mode in one Linac system (Figure 1(c)).

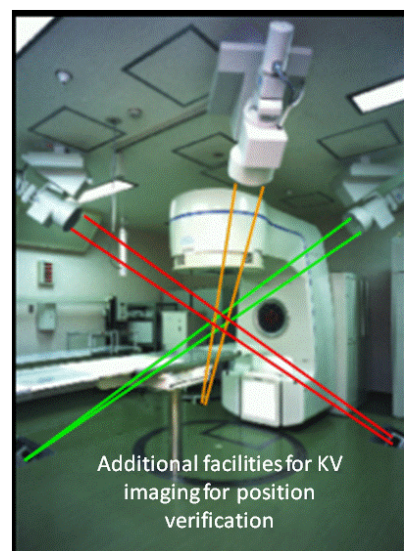

(a)

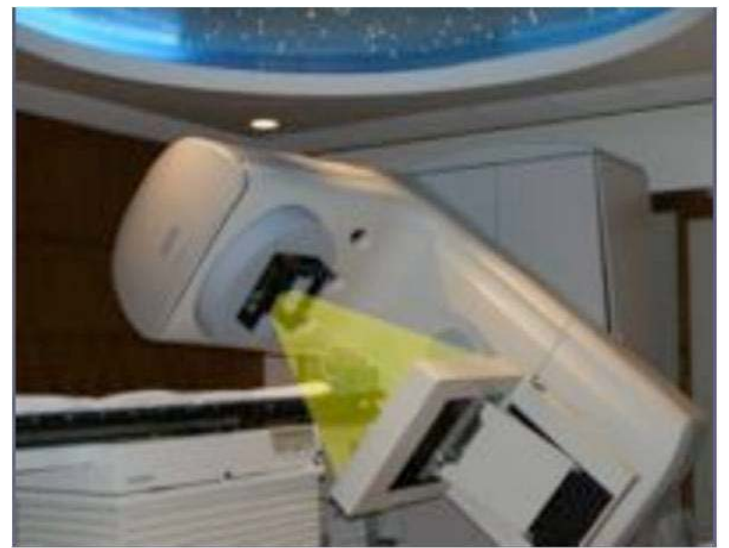

(b)

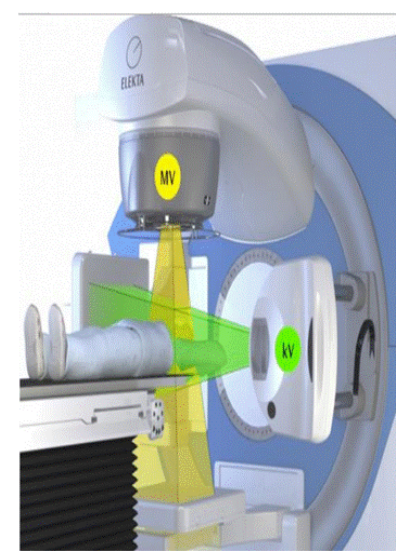

(c)

Figure 1. (a) $\mathrm{kV}$ imaging mode acquired with three additional X-ray generators installed on the roof with different angles. (b) MV imaging mode acquired from the EPID below nozzle. (c) Additional facility for $\mathrm{kV}$ imaging mode mounted on gantry and MV imaging mode from the bottom-imaging detector. 
Our study proposes a high megavoltage-imaging mode (HMV mode) for IGRT. In the HMV mode, X-ray photon energy is bigger than $15 \mathrm{MeV}$, or nominal acceleration potential is greater than $25 \mathrm{MV}$. The HMV mode improves the contrast and quality of imaging as well as not requires additional X-ray generator and control parts as being used in the $\mathrm{kV}$ imaging mode. Therefore, the HMV mode is possible to preserve all of benefits of $\mathrm{kV}$ and MV modes as well as remove the obstacles of size, cost and complexity of $\mathrm{kV}$ mode.

\section{Materials and Methods}

\subsection{Theoretical Basis and Method}

The basic principle of X-ray imaging is the amount of efficient energy that deposited or absorbed in the medium. The more deposited or absorbed energy, the better quality of images will be. The larger differences of the absorbed or deposited energy, the higher capability distinguish these tissues. In other words, it is easier to identify and distinguish the tissue component.

There are three basic forms of the X-ray or photon interacting with mediums. The first one called Photoelectric Effect (PE), which the photon energy is generally lower than $200 \mathrm{keV}$. The second one is Compton Effects (CE), which the photon energy is about $0.2-5.0 \mathrm{MeV}$ and the last one named Electronic Pair Effect (EPE), which the photon energy is larger than $5.0 \mathrm{MeV}$.

The reaction cross-section $\sigma$, in $\mathrm{cm}^{-2}$, is the parameter describes area of the reaction probability for X-ray interacting with medium or matter. The larger cross-section, the more effective energy can be deposited or absorbed in imaging detector, therefore the more benefit to the quality of image.

Equations among the cross-section $\sigma$, the atomic number $Z$ of the matter and the $\mathrm{X}$-ray energy $(h v)$ describe below.

\subsubsection{Theoretical Basis and Method}

$$
\sigma_{t}=\frac{z^{n}}{(h v)^{3}}
$$

where $\sigma$ is the cross-section, the subscript $\tau$ represents Photoelectric Effect. The parameter $z$ is atomic number, $\mathrm{n}$ ranges from 3 to 4 and $h v$ is the energy of photon. The PE is a dominant reaction for $\mathrm{kV}$ imaging mode. From this equation, when X-ray interacting with medium, the reaction cross-section is inversely proportional to the cubic of the photon energy $(h v)$. The smaller of the photon energy and the larger atomic number of medium both lead to the larger scale of the cross-section, and therefore the better of the image quality will be.

\subsubsection{Cross-Section for the Compton Effect}

$$
\begin{aligned}
e^{\sigma} & =2 \pi \int_{0}^{\pi} \frac{\mathrm{d} e^{\sigma}}{\mathrm{d} \Omega_{\theta}} \sin \theta \mathrm{d} \theta \\
& =2 \pi r_{e}^{2}\left\{\frac{1+\alpha}{\alpha^{2}}\left[\frac{2(1+\alpha)}{1+2 \alpha}-\frac{\ln (1+2 \alpha)}{\alpha}\right]+\frac{\ln (1+2 \alpha)}{2 \alpha}-\frac{1+3 \alpha}{(1+2 \alpha)^{2}}\right\}
\end{aligned}
$$

where $\alpha$ is the ratio between the incident X-ray energy ( $h v)$ and electron static energy $\left(m_{e} c^{2}\right)$ which equal 0.511 $\mathrm{MeV}$. Parameter $\theta$ is scattering angle of the scattered photons. $r_{e}$ is a classical radius of the electron. From Equation (2), the cross-section of $\mathrm{CE}$ is independent of the atomic number of medium and the energy of X-ray or photon.

\subsubsection{Cross-Section for the Electronic Pair Effect}

$$
\begin{aligned}
& \text { While } h v>2 m_{e} c^{2}, \sigma_{p} \infty Z h v \\
& \text { While } h v \gg 2 m_{e} c^{2}, \sigma_{p} \infty Z^{2} \ln (h v)
\end{aligned}
$$

where $h v$ represents the energy of X-ray, $m_{e} c^{2}$ is the electronic static energy, $\sigma_{p}$ is cross-section of EPE, $Z$ is the atomic number of the medium. From Equations (3) and (4), the more irradiate X-ray's energy and the bigger scale 
of the cross-section both lead to the better quality of images.

In practical work, we commonly use mass attenuation coefficient $(\mu / \rho)$ rather than the cross-section $\sigma$ with the units of $\mathrm{m}^{2} / \mathrm{kg}$ or $\mathrm{cm}^{2} / \mathrm{g}$ to describe the probability of X-ray photon interacts with medium in unit thickness. The subscript $\mathrm{p}$ represents the effect of EPE. The equation between $\mu_{p} / \rho$ and the medium cross-section $\sigma$ is:

$$
\frac{\mu_{p}}{\rho}=\frac{N_{A}}{M_{A}} \sigma_{p}
$$

In which, $\mu_{p} / \rho$ is the mass attenuation coefficient of X-ray interacting with medium during the period of EPE; $N_{A}$ is Avogadro constant; $M_{A}$ is the Moore mass of the medium.

Accordingly, $\mu_{p} / \rho$ also fully satisfies the following equations:

$$
\begin{aligned}
& \text { if } h v>2 m_{e} c^{2}, \frac{\mu_{p}}{p}=\frac{N_{A}}{M_{A}} \sigma_{p} \infty Z h v \\
& \text { if } h v>m_{e} c^{2}, \frac{\mu_{p}}{p}=\frac{N_{A}}{M_{A}} \sigma_{p} \infty Z \ln (h v)
\end{aligned}
$$

From above theories and formulas, Figure 2 illustrates region distribution of relative predominate with the three main forms of photon interaction with matter corresponding to the function of photon energy and atomic number [11]. The relationship between the mass attenuation coefficient and the photon energy are shown in Figure 3 [12].

If the mass attenuation coefficients of the body tissue, such as bone $Z=12.31$, muscle $Z=7.64$ and fat $Z=6.46$, are normalized with the air mass attenuation coefficient, we can acquire the relative mass attenuation coefficients curve which shown in Figure 4 [13]. Figure 4 shows PE is a predominant absorption mode when the energy ranges from $10-200 \mathrm{keV}$ and named $\mathrm{E} 1$ range. As the PE is proportional to the cubic of atomic number and is inversely proportional to the cubic of the photon energy, these leads to the large absorption energy differences among bone, muscle and fat. As the energy increasing, this energy absorption difference rapidly decreases because CE slowly replaces the PE.

As the CE is a predominant energy absorption mode between $200.0 \mathrm{keV}-10.0 \mathrm{MeV}$ energy and named E2 range, the energy absorption differences of these three mediums are almost disappear. The energy absorption curve nearly is a straight line, which shown as the middle section of Figure 4, because the CE is almost independent of X-ray energy and atomic number of the medium.

In $10.0 \mathrm{MeV}-100.0 \mathrm{MeV}$ energy domain, which named E3 range, the PE and CE completely disappear and the EPE predominate. Therefore, the mass attenuation coefficients of three types of human tissue are proportional

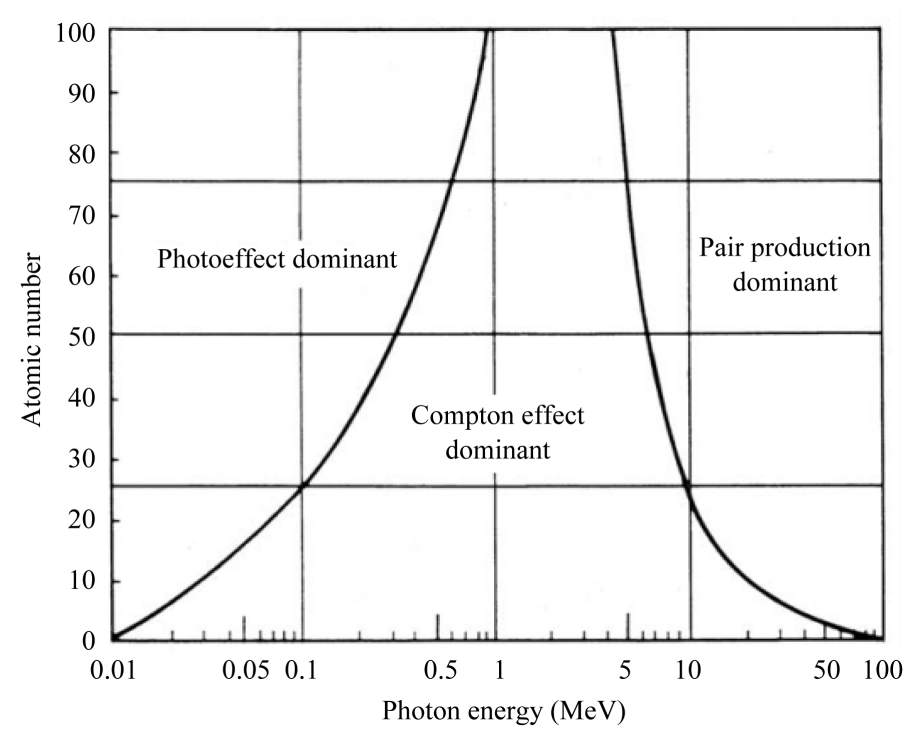

Figure 2. The relation among three interaction forms from photon and medium, photon energy and medium anatomic number. 


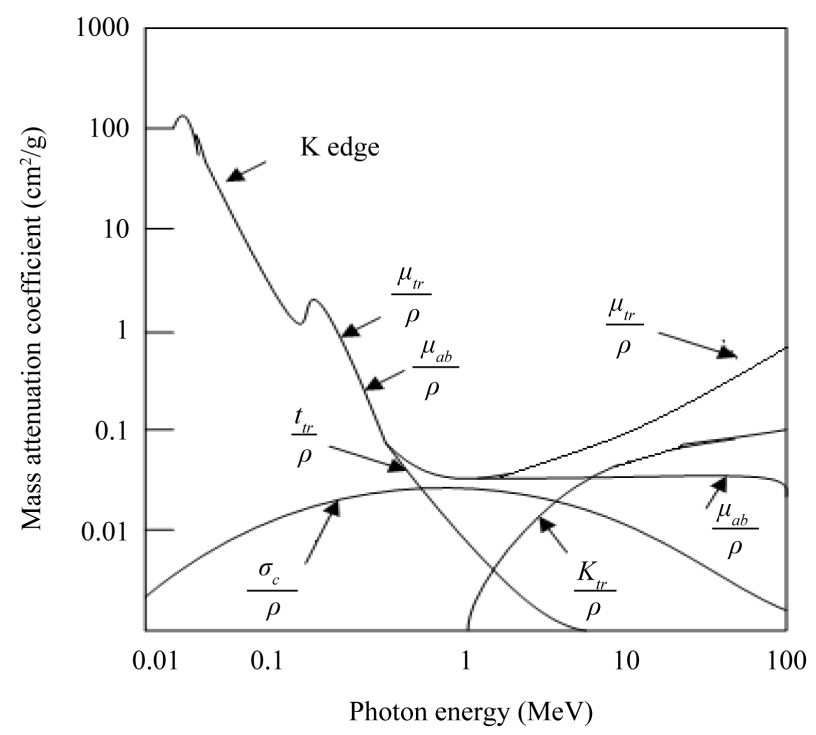

Figure 3. The relationship between mass attenuation coefficient and photon energy.

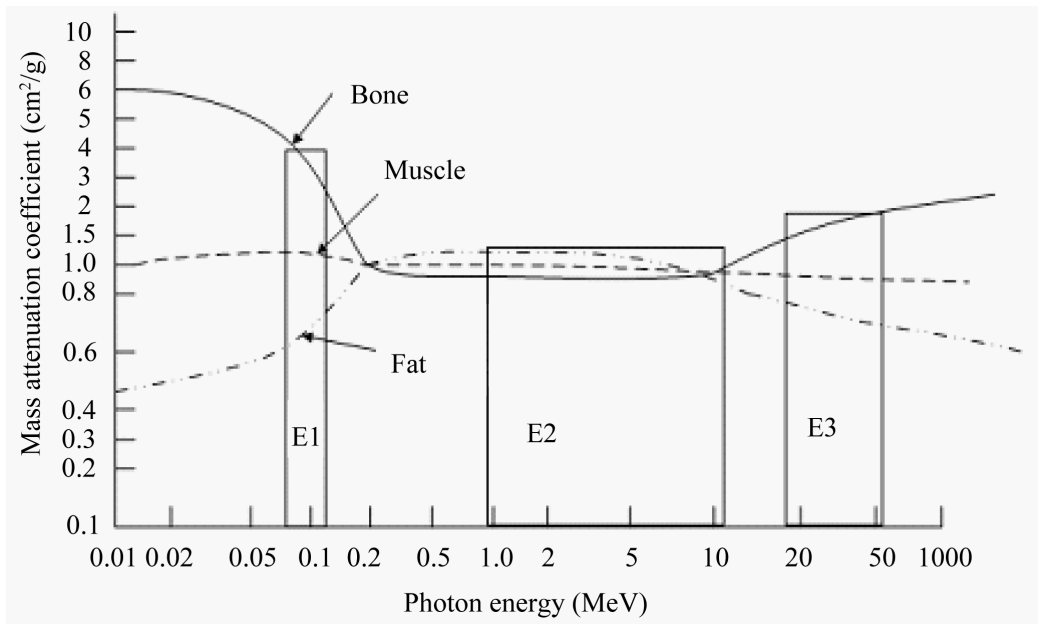

Figure 4. The several of mass attenuation coefficients for bone, muscle and fat with different photon energies. In this figure, E1 represents the Photoelectric Effect becoming a main absorption mode at the ranges of photon energy from $60 \mathrm{keV}$ to 120 $\mathrm{keV}$. E2 means the Compton Effect predominating at the range of photon energy between $1.0 \mathrm{meV}$ to $10 \mathrm{MeV}$. E3 expresses the Electronic Pair Effect becoming a main absorb mode when photon energy ranges from $20 \mathrm{MeV}$ to $50 \mathrm{MeV}$.

to the atomic number $Z$ and the photon energy $h V$ and result in increasing bone absorption. The mass attenuation coefficients differences among three human tissues are also significant. When the photon energy is higher than 15 $\mathrm{MeV}$, this phenomenon becomes much more apparent.

In the $\mathrm{kV}$ image mode, tube voltage of the X-ray generator ranges from $60 \mathrm{kV}$ to $120 \mathrm{kV}$; its bremsstrahlung photon energy approximately equals to $40 \mathrm{keV}$ to $80 \mathrm{keV}$. At this time, the energy range positions in the $\mathrm{E} 1$ of Figure 4 and PE dominates in this region. In addition, at the lower energy value of the energy domain, according to Equation (1), the lower energy leads to the larger cross section area. The detector sensitivity would increase, thereby decreasing noise and increasing the detectability. However, as the energy continue increasing, the crosssection area become decreasing. The detector sensitivity would decrease as well, thereby it leads to increase noise and decrease detectability. Although, there is a decreasing detectability trend within the E1, their differences for the three mediums (bone, muscle and fat) are still huge (as shown in Figure 3 and Figure 4). This is the reason why images quality and the contrast between various tissues and different anatomical structures are both good in 
$\mathrm{kV}$ image mode.

In the MV image mode, the nominal acceleration potential ranges from 4MV to $15 \mathrm{MV}$ and its bremsstrahlung energy approximately equal to $200 \mathrm{keV}$ to $10 \mathrm{MeV}$. At this moment, the CE majorly dominates in this imaging mode. The mass attenuation coefficient in this region is the lowest (as shown in Figure 3 ) and their difference of various tissues and anatomical structures is almost zero (as shown in Figure 4). In addition, as MV energy increases, the exit dose and detector sensitivity would decrease, thereby lead to increase noise and lower detectability. Consequently, the image quality and contrast or resolving capability among these tissues and anatomical structures are the lowest.

According to the above-mentioned theory and analysis, HMV locates in E3 range of Figure 4. EPE dominates in this region. In comparison, the mass absorption coefficient is larger and the difference of X-ray absorption coefficient among three tissues (bone, muscle and fat) is much more considerable than the difference has been found in the MV imaging mode (see Figure 3). In addition, as HMV energy increases, the exit dose and detector sensitivity would increase, thereby decreasing noise and increasing detectability. So this mode not only offers better images quality, but also gains better contrast or resolving capability between tissues and anatomical structures than conventional MV imaging mode.

\section{Experiment}

All experiments have been done by using The linear accelerator (LA 45, Top Grade Healthcare, Beijing, China), shown in Figure 5. The incident X-ray energy that used for approving previous theoretical statements was $10 \mathrm{MV}$, $25 \mathrm{MV}$ and $45 \mathrm{MV}$ bremsstrahlung, which was equivalent to $8 \mathrm{MeV}, 13 \mathrm{MeV}$ and $30 \mathrm{MeV}$ X-ray energy respecttively [14].

PekinElmer, a company in Germany, provided X-ray imaging system. The X-ray flat panel detector used in experiments was an indirect conversion type and consisted of amorphous silicon. The CsI (Tl) scintillator (PekinElmer, Germany) with XIS3.2.0.67 image acquisition and analyzing software also included in the panel. The series number of the X-ray panel detector is XRD 1640 AN19 CS. The flat panel's physical dimension is " $16 \times 16$ " with the precise of $200 \mu \mathrm{m}$ (nominal spatial resolution $2.5 \mathrm{lp} / \mathrm{mm}$ ). The original projection acquired from flat panel was 16 bit (or 65,000 grayscale) and had a resolution of $1024 \times 1024$ pixels.

The image detector panel (Figure 6(a)) placed under the treatment head of the LA45 accelerator. The distance between flat panel's surface and X-ray source was $1480 \mathrm{~cm}$. The center of the medium (phantom or patient) that need to be scanned located at isocenter of LA45. The dose of single expose was $2 \mathrm{cGy}$ each time, took $600 \mathrm{~ms}$ acquisition time for collecting three frame images and chose the best quality one.

The phantom used in the experiments was the Catphan 500 (Figure 6(b)) cylinder phantom (acquired from The Phantom Laboratory, NewYork, USA), which was mainly used for evaluating the scanning performance of CT or CTBT as consisting of several material modules in different density and computed tomography (CT) value (range from $-1000 \mathrm{Hu},-200 \mathrm{Hu},-100 \mathrm{Hu},-35 \mathrm{Hu}, 120 \mathrm{Hu}, 340 \mathrm{Hu}, 990 \mathrm{Hu}$ ) [15]. In this study, the phantom used for evaluating image quality of FPD with different X-Ray's energy irradiation (10 MV, $25 \mathrm{MV}$ and $45 \mathrm{MV}$ ), where 10 MV stands for MV imaging mode and $45 \mathrm{MV}$ stands for HMV imaging mode. Based on this experiment's result, a real patient's abdomen was also be scanned with the same method. All images were acquired by using screenshot in high-resolution medical displayer and were processed with Paint tool from Windows software.

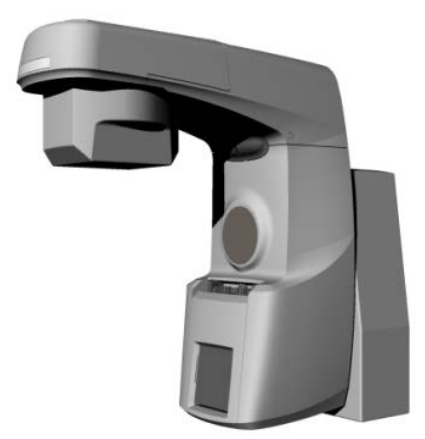

Figure 5. The LA45 linear accelerator used in this experiment. 


\section{Results}

The images of the Catphan 500 phantom from $10 \mathrm{MV}, 25 \mathrm{MV}$ and $45 \mathrm{MV}$ with 2cGy high energy bremsstrahlung irradiation are respectively shown in Figures 7(a)-(c). Figure 8(a) is a verification image that was acquired from the world famous accelerator with 6 MV X-ray IGRT system of a patient's abdomen image. Figures 8(b)-(d) are abdomen images of the same patient with 10, 25, $45 \mathrm{MV}$ for 2cGy high energy bremsstrahlung irradiation, respectively.

\section{Discussions}

Obviously, the image quality and the resolution of 45 MV bremsstrahlung irradiation on either Catphan 500 phantom or patients has better resolution than the image acquired of $25 \mathrm{MV}$ bremsstrahlung. The latter is also superior to each of image acquired from $10 \mathrm{MV}$ and $6 \mathrm{MV}$, respectively. This result conformed to the statements that derived from theoretically analyzing in previous sections. The advantages of using HMV image mode were more apparent than applying conventional $\mathrm{kV}$ and MV imaging mode. If using high-resolution medical displayer directly analyzes these images, the advantages of HMV would be much more apparent.

Theoretical and experimental results show that HMV image mode not only improves the image quality, but also has good resolving capability to identify tissues components. As the detector placed below treatment head and shares the same source of treatment, HMV imaging mode prevents drawbacks of position verification from both $\mathrm{kV}$ and MV level imaging mode as well as keeps their advantages.

\section{Conclusion}

In conclusion, HMV is a good imaging verification mode in radiotherapy; it will play a sensible role in radio-

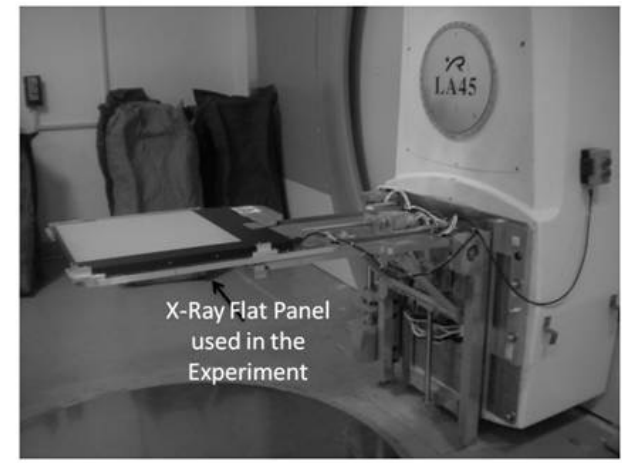

(a)

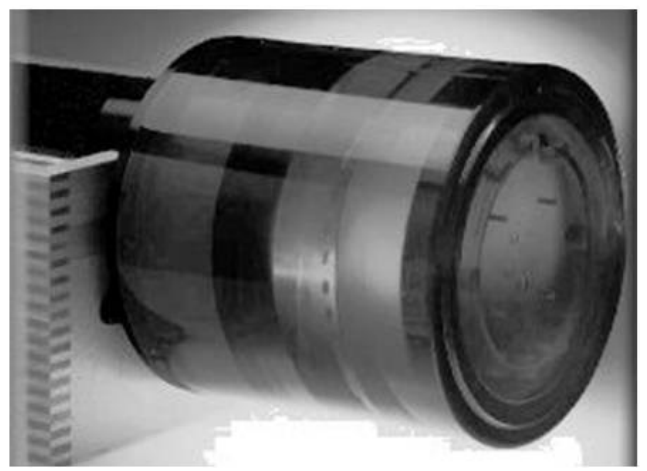

(b)

Figure 6. (a) X-ray flat panel detector used in this experiment; (b) Catphan 500 cylinder phantom.

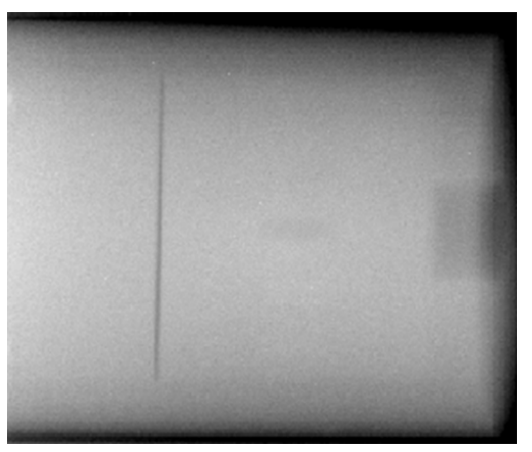

(a)

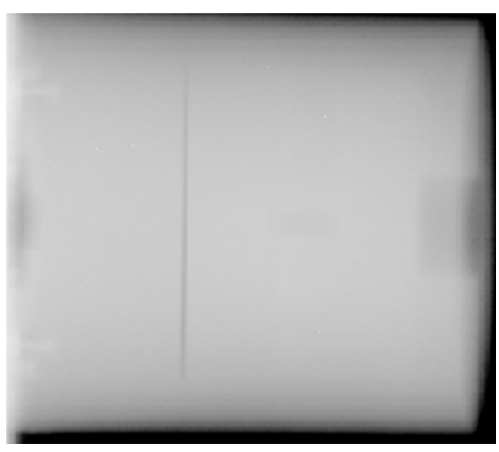

(b)

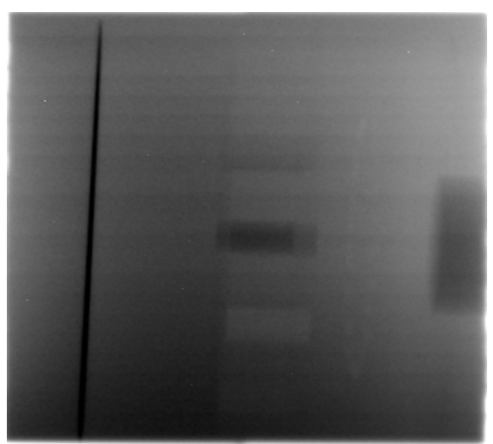

(c)

Figure 7. Comparison with different bremsstrahlung energies irradiated on the Catphan 500 phantom. The phantom is irradiated by $10 \mathrm{MV}$ bremsstrahlung (a), $25 \mathrm{MV}$ bremsstrahlung (b) and $45 \mathrm{MV}$ bremsstrahlung (c), respectively. The main difference of these images is also pointed. 


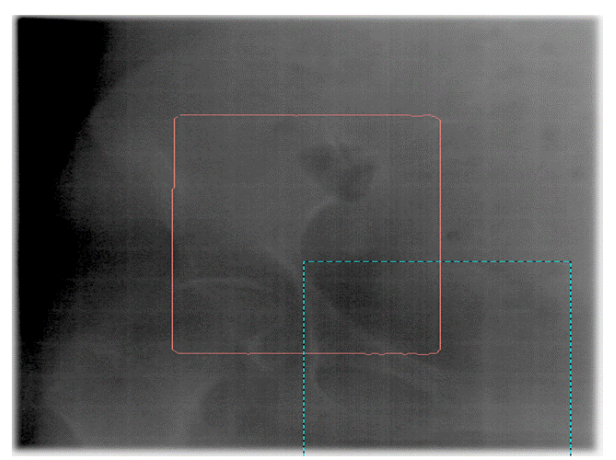

(a)

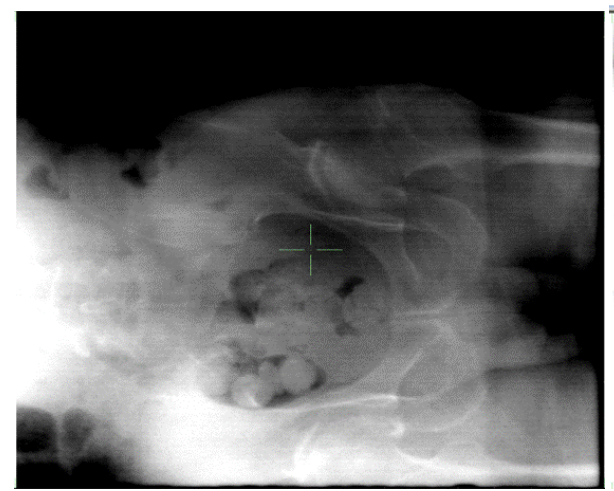

(c)

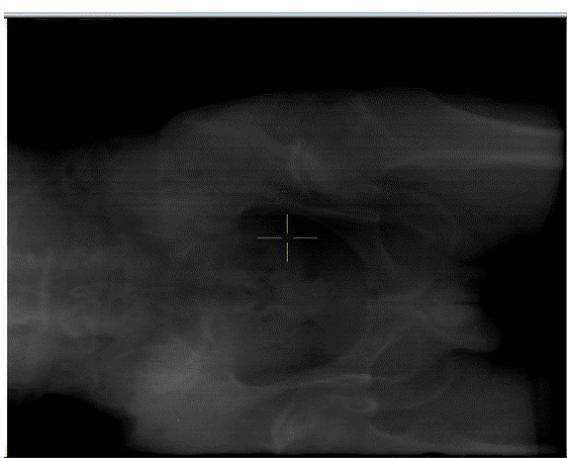

(b)

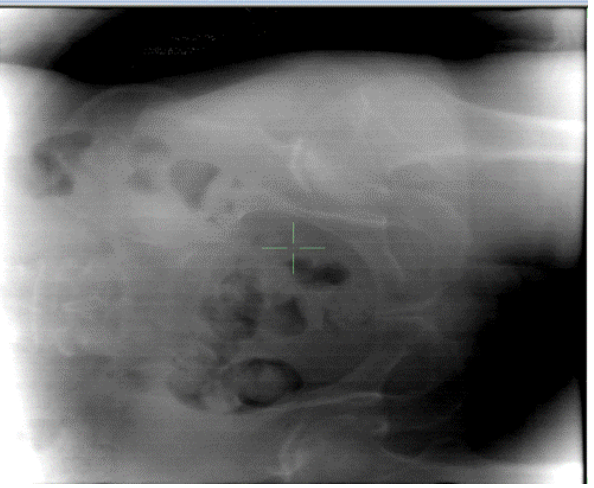

(d)

Figure 8. Abdomen images of the same patient from various bremsstrahlung X-ray energies compare to conventional $6 \mathrm{MV}$ X-ray IGRT. (a) $6 \mathrm{MV}$ radiation image from the world famous accelerator; (b) abdomen was irradiated with $10 \mathrm{MV}$ bremsstrahlung; (c) abdomen was irradiated with $25 \mathrm{MV}$ bremsstrahlung and (d) abdomen was irradiated with $45 \mathrm{MV}$ bremsstrahlung.

therapy position verification.

\section{References}

[1] Hurkmans, C.W., Cho, B.C., Damen, E., Zijp, L. and Mijnheer, B.J. (2002) Reduction of Cardiac and Lung Complication Probabilities after Breast Irradiating Using Conformal Radiotherapy with or without Intensity Modulation. Radiation Oncology, 22, 163-171. http://dx.doi.org/10.1016/S0167-8140(01)00473-X

[2] Jacob, R., Hanlon, A.L., Horwitz, E.M., Movsas, B., Uzzo, R.G. and Pollack, A. (2004) The Relationship of Increasing Radiotherapy Dose to Reduced Distant Metastases and Mortality in Men with Prostate Cancer. Cancer, 100, 538-543. http://dx.doi.org/10.1002/cncr.11927

[3] Chen, J., Morin, O., Aubin, M., Bucci, M.K., Chuang, C.F. and Pouliot, J. (2006) Dose Guided Radiation Therapy with Megavoltage Cone Beam CT. The British Journal of Radiology, 79, S87-S98. http://dx.doi.org/10.1259/bjr/60612178

[4] Jaffray, D.A., Siewerdsen, J.H., Wong, J.W. and Martinez, A.A. (2002) Flat Panel Cone-Beam Computed Tomography for Imaging Guided Radiation Therapy. International Journal of Radiation Oncology, Biology, Physics, 53, 1337-1449. http://dx.doi.org/10.1016/S0360-3016(02)02884-5

[5] Pouliot, J., Bani-Hashemi, A., Chen, J., Svators, M., Ghelmansarai, F., Mitschke, M. and Xia, P. (2005) Low-Dose Megavoltage Cone-Beam CT for Radiation Therapy. International Journal of Radiation Oncology, Biology, Physics, 61, 552-560. http://dx.doi.org/10.1016/j.ijrobp.2004.10.011

[6] Groh, B.A., Siewerdsen, J.H. and Drakea, D.G. (2002) A Performance Comparison of Flat-Panel Imager-Based MV and kV Cone-Beam CT. Medical Physics, 29, 967-975. http://dx.doi.org/10.1118/1.1477234

[7] Ruchala, K.J., Olivera, G.H., Schloesser, E.A. and Mackie, T.R. (1999) Megavoltage CT on a Tomotherapy System. Physics in Medicine and Biology, 44, 2597-2621. http://dx.doi.org/10.1088/0031-9155/44/10/316

[8] (2015) Personal Conversation with Experts at the American Association of Physicists in Medicine (AAPM). 
[9] Murphy, M.J., Balter, J., Balter, S., Bencomo, J.A., Das, I.J., Jiang, S.B., Ma, C.M., Olivera, G.H., Rodebaugh, R.F., Shirato, H. and Yin, F.F. (2007) The Management of Imaging Dose during Image-Guided Radiotherapy: Report of the AAPM Task Group 75. Medical Physics, 34, 4041-4060. http://dx.doi.org/10.1118/1.2775667

[10] Liu, L., Antonuk, L.E., EI-Mohri, Y., Zhao, Q.H. and Jiang, H. (2015) Theoretical Investigation of the Design and Performance of a Dual Energy (kV and MV) Radiotherapy Imager. Medical Physics, 42, 2072-2084. http://dx.doi.org/10.1118/1.4915120

[11] Thilmann, C., Nill, S., Tứcking, T., Hoss, A., Hesse, B., Dietrich, L., Bendl, R., Rhein, B., Häring, P., Thieke, C., Oelfke, U., Debus, J. and Huber, P. (2006) Correction of Patient Positioning Errors Based on In-Line Cone Beam CTs: Clinical Implementation and First Experiences. Radiation Oncology, 24, 1-16.

[12] Podgorsak, E.B. (2005) Radiation Oncology Physics: A Handbook for Teachers and Students. International Atomic Energy Agency, Vienna.

[13] Hu, Y.M. (1999) The Tumor Radiation Physics. Atomic Energy Pressed, Beijing.

[14] Zhang, Q.S., Zhang, M. and Wu, X.Y. (2005) Research on Radiation Quality and Scanning Beam Characteristics of Medical Electron Cyclotron. Chinese Journal of Medical Physics, 22, 376-378.

[15] Guan, H.Q. and Dong, H. (2009) Dose Calculation Accuracy Using Cone-Beam CT for Pelvic Adaptive Radiotherapy. Physics in Medicine and Biology, 54, 6239-6250. http://dx.doi.org/10.1088/0031-9155/54/20/013 